\title{
Proposal and Evaluation of APIs for utilizing Inter-Core Time Aggregation Scheduler
}

\author{
Satoshi Yamada and Shigeru Kusakabe \\ Graduate School of Information Science and Electrical Engineering, \\ Kyushu University, 744, Motooka, Nishi-ku, Fukuoka, Japan \\ satoshi@ale.csce.kyushu-u.ac.jp \\ kusakabe@ait.kyushu-u.ac.jp
}

\begin{abstract}
This paper proposes and evaluates APIs for Inter-Core Time Aggregation Scheduler (IAS), which is a kernel-level thread scheduler to enhance performances of multi-threaded programs on multi-core processors. We have proposed IAS, which is a combination of time-multiplexing and space-multiplexing scheduling to utilize caches existing per processing core and shared between processing cores.

We present the effect of APIs in two aspects. Firstly, we show that we can effectively and easily set the aggregation strength in IAS based on the quantum time, which is a period of time that a thread uses CPU. Secondly, we show that we can gain the effect of space-multiplexing without setting processor affinity of each thread by splitting processing cores into several groups and running IAS per group. We implement IAS and its APIs by modifying a Linux kernel and present its effect on a commodity multi-core processor.
\end{abstract}

Key words: Thread Scheduling, Multi-core Processor, Cache Sharing, Multi-threaded Program

\section{Introduction}

In this paper, we show the proposal and the evaluation of APIs for Inter-Core Time Aggregation Scheduler (IAS). IAS is a kernel-level thread scheduler to enhance the performance of multi-threaded programs on a commodity multicore processor. IAS is a combination of time-multiplexing and space-multiplexing scheduler to utilize the caches existing per processing core (Core) and shared between Cores. The contribution of this paper is as follows:

- We show that we can effectively and easily set the aggregation strength in IAS based on the quantum time, which is a period of time that the thread uses CPU.

- We show that we can gain the effect of space-multiplexing without setting the processor affinity of each thread by splitting Cores into several groups and running IAS per group. 
Nowadays, we have many kinds of multi-core processors, such as Simultaneous Multi-Threading (SMT), Chip Multi-Processing (CMP), and Chip MultiThreading (CMT), where we can execute threads in parallel. One of the main differences between multi-core processors and conventional shared-memory multiprocessors is that caches, typically L2 caches, are generally shared by Cores in multi-core processors. It is widely known that combinations of threads running simultaneously on different Cores affect the utilization of caches and the performance because Cores compete the shared cache with each other in a multi-core processor [1-3]. To utilize the shared cache in a multi-core processor, we propose a thread scheduling mechanism which focuses on multi-threaded programs.

In this paper, a multi-threaded program means a program executing multiple kernel-level threads sharing the same memory address space in parallel. In Linux, for example, we can implement multi-threaded programs with POSIX library, Java, Perl, MPI, OpenMP, and Open64 because a thread in these languages and compilers systems corresponds to a native thread in the kernel. The rationale of focusing on only multi-threaded programs is that many modern programs, especially commercial programs, are getting multi-threaded as multi-core processors widely spread. For example, database servers and Web servers, such as MySQL and Apache HTTP Server, are multi-threaded to handle multiple client connections efficiently. The modern benchmark programs such as DaCapo benchmarks[4] and Parsec Benchmark[5] also employ multi-threading to simulate the popular and emerging workloads. We expect that we will have more multi-threaded programs and more chances to apply our scheduling mechanism.

We show the overview of our scheduling mechanism in Fig. 1. The scheduling mechanism is made up of three domains, Kernel, User, and Helper-thread. Kernel domain provides a basic scheduling mechanism and implemented as IAS. User and Helper-thread are domains which control the parameters for Kernel domain. User domain provides the interfaces to control the parameters explicitly assuming that users are aware of the characteristics of the workloads. Helper-thread domain analyzes the characteristics of the currently executed workloads, detect the degradation of the performance of multi-threaded programs, and controls the parameters autonomically. Thus, our scheduling mechanism can adopt to the characteristics of the workloads without modifying and re-building Kernel. In this paper, we focus on User domain, and present the APIs to control the behavior of IAS while Helper-thread domain is our future work.

IAS is a kernel-level thread scheduler for commodity platforms with multicore processors and implemented by modifying a Linux kernel. IAS dynamically aggregates sibling threads, kernel-level threads sharing the same memory address space, and executes them simultaneously on different Cores based on the assumption that sibling threads share a certain amount of working set, the memory area to be accessed by threads. The benefit of IAS is to increase the possibility that co-scheduled threads share their working set and decrease the capacity pressure on the cache. IAS may increase the simultaneous access to the working set, where only transactional access is permitted with locks and semaphores, and cause frequent stalls. However, according to the researches on the analysis of the 
performance of CMP, the L2 cache misses caused by the lack of capacity are the most influential $[3,6]$. Therefore, we expect the enhancement of the performance by IAS.

Previously, we investigated the effect of IAS with several multi-threaded benchmark programs and clarified two problems for the effective use of IAS[7, 8]. The first problem is the aggregation strength. The effect of IAS depends on the characteristic of programs such as the size of shared working set between sibling threads, and platforms such as shared cache size. In case sibling threads share working set, strong aggregations of sibling threads are likely to enhance the performance. On the other hand, IAS can degrade the performance when the workload is $\mathrm{I} / \mathrm{O}$ intensive and the aggregation of sibling threads results in poor utilization of CPU. For this reason, we should control if we should aggregate sibling threads of a program or not, and the aggregation strength. The second problem is the combinations of Cores to execute IAS. IAS aggregates sibling threads on the combination of Cores specified in the kernel. Previously, we have evaluated the effect of IAS on a dual-core processor. In the dual-core processor, we can make only a single combination of Cores. Nowadays, the number of Cores has increased and the structure of the memory hierarchy tends to become complex like Intel Core i7. In such platforms, aggregations of sibling threads with a single combination of Cores may increase the overhead of communications between Cores because we assume that sibling threads share a certain amount of working set. Setting processor affinities and isolating Cores for every program like a conventional space-multiplexing may decrease the communication between Cores. However, it is another difficult issue to optimally set the processor affinity of each thread. We consider that setting multiple combinations of Cores to run IAS can reduce the overhead of communications between Cores and we should have an interface to control the combinations.

In this paper, we propose and evaluate APIs for IAS to settle the problems mentioned above. We show that we can effectively and easily set the aggregation strength in IAS based on the quantum time of the previously executed thread. We also show that we can gain the effect of space-multiplexing without setting the processor affinity of each thread by splitting Cores into several groups and running IAS per group.

The rest of this paper is organized as follows. Section 2 explains the implementation and preliminary evaluation of IAS. Section 3 explains the proposal of APIs. Section 4 presents the evaluation of APIs. Section 5 introduces related works and clarifies our research position. We conclude in Section 6 .

\section{Implementation and Evaluation of Inter-Core Time Aggregation Scheduler (IAS)}

In this Section, we explain the implementation and the evaluation of IAS. We implement IAS by modifying Completely Fair Scheduler (CFS) in Linux 2.6.24 because we assume the use of IAS on commodity processors. IAS ignores the inversion of the priority of each thread in SCHED_NORMAL class, which is 


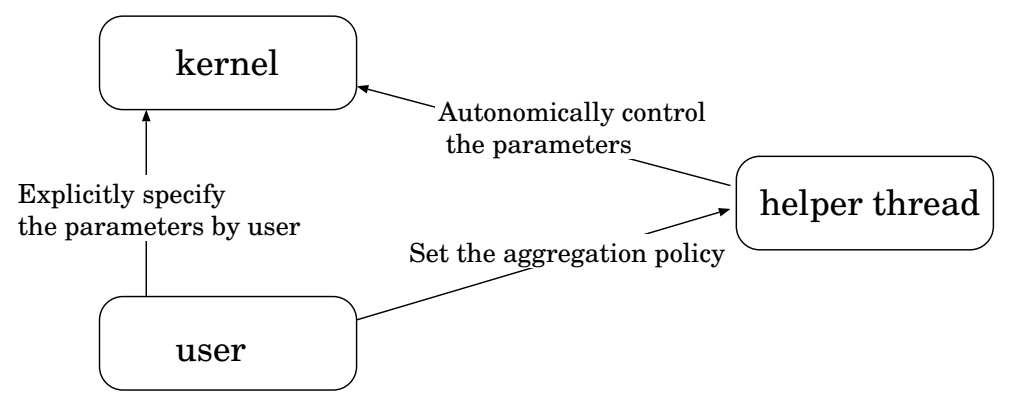

Fig. 1. The overview of the scheduling mechanism. We divide the scheduling functions into three domains, which enables the dynamic and flexible control of thread aggregation. In this paper, we focus on User domain.

non-real-time thread in Linux, and dynamically aggregates sibling threads. We explain the scheduling mechanism of CFS for threads of SCHED_NORMAL class in Section 2.1 and IAS in 2.2. In Section 2.3, we show the preliminary evaluation of IAS on a commodity processor. Based on the preliminary evaluation, we show the problems of running IAS and necessity of effective APIs.

\subsection{Completely Fair Scheduler (CFS)}

CFS is the standard thread scheduler employed in Linux since its version 2.6.23. CFS is designed to equally distribute CPU time to threads with the same static priority. CFS counts the quantum time of each thread in nanoseconds and calculates the priority as vruntime based on the quantum time and nice value. When a thread is dispatched by the scheduler, the additional vruntime is calculated from the quantum time and added to vruntime of the thread. CFS sets higher priority for threads with less vruntime to accomplish the fair usage of CPU between threads which start at the same time with the same nice value. The runqueues and independent schedulers exist per Core. The load balancer in CFS equalizes the sum of weight, which is a value corresponding to nice value and defined in the kernel, between runqueues. CFS does not recognize the memory address space of each thread both in scheduling and load balancing.

\subsection{Overview of Inter-Core Time Aggregation Scheduler (IAS)}

IAS executes two scheduling policies at the same time. The first scheduling policy is the time aggregation, which executes sibling threads in a row on a single Core. The second scheduling policy is the inter-core aggregation, which executes sibling threads on different Cores at the same time. In this section, we firstly explain Time Aggregation Scheduler (TAS), which is the implementation of the time aggregation. Then, we explain the extension of TAS to add the inter-core aggregation. 
Implementation of Time Aggregation Scheduler (TAS) The basic idea of the implementation of TAS is to dynamically give a priority bonus to the sibling thread of the currently executed thread. As we mentioned in Section 2.1, the priority of a thread is higher when vruntime of the thread is smaller. Therefore, the priority bonus for TAS works to reduce vruntime of the sibling thread. To implement this idea in CFS, we add a flag to task_struct, the structure to maintain the states of a thread in Linux, to recognize if the thread has the sibling threads or not. When a thread creates its sibling thread, TAS sets the flag and links the thread with the list of its sibling threads. The list of the sibling threads exists per Core and sorted in the ascending order of vruntime. We show an example case of the time aggregation in Fig. 2.

Fig. 2 shows the runqueue of $\mathrm{CFS}^{1}$ and the additional links of sibling threads for the time aggregation. The circles in Fig. 2 represent threads and the square involving threads represents a runqueue. The numbers in the threads show vruntime of each thread. Each thread in Fig. 2 owns vruntime of around 10 to 20 for ease of explanation, however, it is common for threads in Linux to own vruntime in the millions and the billions calculated from their quantum time counted in nano seconds. Threads are queued in the ascending order of vruntime and shown from the left in the runqueue in Fig. 2. The pattern inside the thread represents the memory address space. Our scheduler links the sibling threads in the ascending order of their vruntime. The links between sibling threads are dashed lines in Fig. 2. We add a member, mm_sibling, to the structure of the memory address space, mm_struct, in Linux. The link of the sibling threads begins with mm_sibling (represented as mm in Fig. 2). The currently executed thread $\mathrm{A}$ has been dequeued from the runqueue. After executing thread A, CFS selects thread B as the next thread. TAS checks if the flag for the sibling threads is set in thread A. TAS recognizes that the flag is set and looks for the sibling thread from the list of the sibling thread starting from the $\mathrm{mm}_{-} \mathrm{sibling}$ of thread A. TAS finds thread $\mathrm{C}$ from the list and considers thread $\mathrm{C}$ as another candidate. We set the priority bonus for aggregating sibling threads in advance and our scheduler calculates the expression below.

$$
\text { B.vruntime }>\text { C.vruntime - priority_bonus }
$$

In this paper, we express vruntime of a thread as thread_ID.vruntime like B.vruntime. If expression (1) is true, then TAS will select thread C. If we set the priority bonus equal to or larger than 7 in Fig. 2, TAS will select thread $\mathrm{C}$ as the next thread. Otherwise, TAS selects thread B. Thus, TAS is able to aggregate sibling threads while considering the priority of each thread. Also, The scheduling algorithm of TAS is $O(1)$ because the link of sibling threads is sorted in ascending order of vruntime.

\footnotetext{
${ }^{1}$ The runqueue of CFS has a structure of Red-black tree. We express the runqueue as a list for ease of explanation.
} 


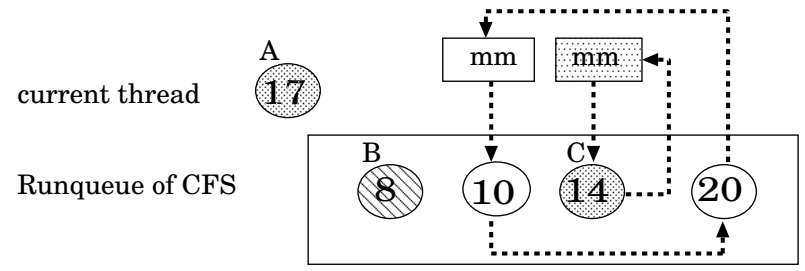

Fig. 2. Example case of TAS. A circle represents a thread and the pattern inside the circle expresses its memory address space. TAS looks for the sibling thread of the current thread from the list of the sibling threads. If there exists a sibling thread (thread C), TAS considers the thread as the candidate for the next thread.

Extension of TAS to add the inter-core aggregation We extend TAS to add the inter-core aggregation to implement IAS. First of all, we run independent TAS per Core and divide each Core into master and slave Cores. IAS lets every Core cooperatively aggregate sibling threads by making slave Cores follow the aggregation on master Core. When the scheduler on master Core finds a chance of aggregating sibling threads, it sets a pointer, ia mm, to the memory address space of the currently executed thread. Otherwise, ia_mm is NULL. Only master Core can manipulate ia mm while slave Cores only refer to ia $\mathrm{mm}$. When ia mm is set to an actual memory address space, the schedulers on slave Cores look for the sibling threads sharing the memory address space, which ia_mm points to, from their own runqueue. If there exists sibling threads, the schedulers consider the threads as the candidates for the next scheduled threads with the priority bonus.

We show an example case of IAS on a platform of a dual-core processor in Fig. 3. In Fig. 3, the circles represent threads and squares represent runqueues on each Core. The pattern inside the thread represents the memory address space and three threads are waiting in the runqueues on each Core. While we omit vruntime values in Fig. 3, threads are enqueued into each runqueue in the ascending order of their vruntime from the left. Thread A is running on master Core and thread $\mathrm{E}$ is running on slave Core. We also omit the links between sibling threads in Fig.3. Thread B on master Core and thread F on slave Core are to be scheduled next to thread A and thread $\mathrm{E}$ in case of CFS. After executing thread $\mathrm{A}$ on master Core, thread $\mathrm{C}$ is also the candidate to be scheduled next because thread $\mathrm{C}$ is the sibling thread of thread $\mathrm{A}$. If thread $\mathrm{C}$ satisfies expression (1), the scheduler on master Core sets the memory address space of thread C to ia mm (solid arrow in Fig. 3). On slave Core, the scheduler checks ia mm in scheduling (dashed arrow in Fig. 3). After executing thread E, thread F, G, and $\mathrm{H}$ are the candidates because thread $\mathrm{G}$ is a sibling thread of thread $\mathrm{E}$ and thread $\mathrm{H}$ is a sibling thread sharing the memory address space set in ia_mm. To execute sibling threads simultaneously on different Cores, IAS enhances the priority of the thread sharing the memory address space, which ia_mm points to, with the priority bonus. Thread $\mathrm{H}$ has the priority bonus against thread $\mathrm{F}$ and against 
thread G. If thread $\mathrm{H}$ satisfies both expression (2) and expression (3), thread $\mathrm{H}$ will be scheduled after thread E.

$$
\begin{aligned}
& \text { F.vruntime }>\text { H.vruntime }- \text { priority_bonus } \\
& \text { G.vruntime }>\text { H.vruntime }- \text { priority_bonus }
\end{aligned}
$$

Following the steps above, IAS can execute sibling threads nearly simultaneously on different Cores while considering the priority of each thread. When thread $\mathrm{H}$ does not satisfy expression (2) and (3), IAS behaves as TAS. If expression (4) is satisfied, thread G will be the next thread. If expression (4) is not satisfied, thread $\mathrm{F}$ will be scheduled.

$$
\text { F.vruntime }>\text { G.vruntime - priority_bonus }
$$

IAS uses the link of sibling threads, which we use for the time aggregation, to search for the sibling threads. The scheduling cost of IAS is also $O(1)$ because the sibling threads are sorted in ascending order in the link.

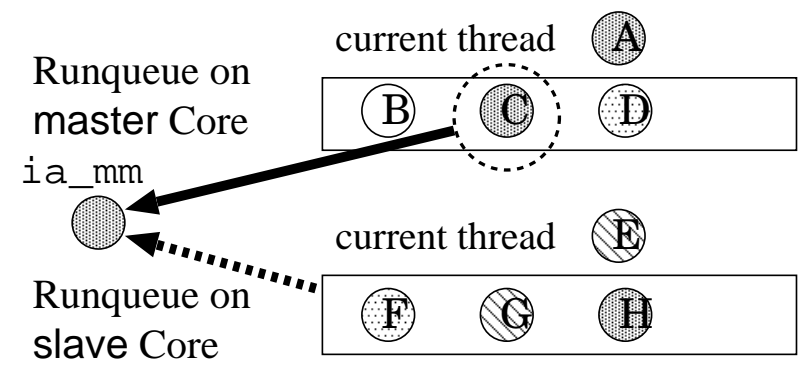

Fig. 3. Example case of IAS. When sibling threads (circles with the same pattern) are aggregated in Core 0 by TAS, the memory address space of the sibling thread is registered in ia_mm. The scheduler on Core 1 looks for threads sharing the same memory address space with ia_mm and considers the thread as the candidate for the next thread.

\subsection{Preliminary Evaluation of Inter-Core Time Aggregation Scheduler (IAS)}

In this section, we show the preliminary evaluation of IAS in terms of its overhead against CFS. We also show the effect of IAS on RUBiS benchmark[9], which is a benchmark program to measure the performance of a Web application server running a multi-threaded HTTP server and a database server simultaneously. Firstly, we show that the overhead of IAS is trivial compared to CFS. Then, we show that the effect of IAS depends largely on the value of the priority bonuses[8], indicating that an easy and effective way of controlling the priority bonus is necessary. 
Overhead of IAS We evaluate the additional overhead of IAS compared to CFS. The following tasks are the overhead of IAS.

- Setting the flag of sibling threads in the added member of task_struct

- Setting link between sibling threads in the runqueues

- Considering sibling threads in scheduling

We implement a benchmark, which measures the execution time of creating and joining multiple sibling threads, to evaluate the total additional overhead of IAS. The created sibling threads just join to the parent thread. Also, we set the priority bonus for IAS as 0 to schedule threads according to the priority of CFS. We consider that we can purely measure the sum of the listed overhead by comparing the execution time in CFS and IAS.

According to our measurements, we see the increase of the execution time in IAS by $1 \%$ in creating and joining $500 \mathrm{~K}$ sibling threads. We consider that the overhead is small enough to be implemented in CFS. In case the aggregation of sibling threads degrade the performance, we only have to set the priority bonus as 0 .

Effect of IAS on RUBiS benchmark We show the effect of IAS in running RUBiS benchmark in Table 1. RUBiS is a benchmark application which simulates the workload of ebay.com and evaluates the performance of a Web application consisting of a HTTP and a database server. RUBiS sends simultaneous requests from multiple clients to the Web application server and evaluates the throughput (Completed Sessions) and the average response time (Response Time) of each request. Both HTTP (Apache HTTP server 2.2.8) and database servers (MySQL 5.0.45) are multithreaded, therefore, IAS aggregates threads of both servers. We use RUBiS benchmark because each thread of these transaction-oriented applications is likely to share the working set rather than scientific application benchmark[10,11]. We change the value of the priority bonus and compare the result with CFS. The numbers in the parentheses indicates the ratio of the result in IAS against CFS.

In Table 1, we see the increase of the throughput and the reduction of the response time in IAS compared to CFS, indicating IAS is effective in enhancing the performance of a Web application server. We also see that the effect varies as we change the priority bonus and we have to set the priority bonus around 10 millions to maximize the effect. When we set the priority bonus as high as 100 millions vruntime, IAS aggregates too many sibling threads of one server and let the sibling threads of another server wait too long. The result shows that we have to tune the priority bonus to accomplish the optimal performance in running multiple multi-threaded programs.

\subsection{Problems of IAS}

Based on the preliminary evaluation in Section 2.3, we present two problems in running IAS as shown below. 
Table 1. Result of RUBiS benchmark

\begin{tabular}{l|r|r|r|r}
\hline Kernel & CFS & \multicolumn{3}{|c}{ IAS } \\
\cline { 3 - 5 } & & 1Mvruntime & 10Mvruntime & 100Mvruntime \\
\hline \hline Completed Sessions & 230 & $259(1.12)$ & $301(1.30)$ & $265(1.15)$ \\
\hline Response Time (ms) & 62,556 & $48,760(0.77)$ & $43,090(0.68)$ & $53,230(0.85)$ \\
\hline
\end{tabular}

- Control of the priority bonus

- Allocation of master/slave

Firstly, the effect of IAS depends on the characteristic of each program. In case IAS degrades the total performance by the aggregation of some programs, users should have an interface to set the priority bonus as 0 or tell the kernel not to aggregate the sibling threads of those programs. Even when IAS enhances the total performance by aggregating the sibling threads of some programs, the priority bonus should be given in proper strength to maintain the fairness of CPU usage between threads. Assuming users are aware of the characteristics of each program in advance, it is still difficult to properly give the priority bonus in vruntime. As we mention in Section 2.1, vruntime is calculated in the order of nano seconds and too fine-grained for users to control. We consider that users should have an interface to control the aggregation strength other than specifying the priority bonus in vruntime.

Secondly, users should have an interface to allocate multiple master/slave groups flexibly. Nowadays, we have many Core processors with complex memory hierarchy. For example, Intel Core 2 Quad has four Cores. Each Core has own L1 data/instruction cache and a single L2 cache is shared between two Cores. There is no cache shared between every Core. In this case, aggregating sibling threads with a single ia_mm may increase the overhead of communication between Cores not sharing the same L2 cache. We consider that users should have an interface to allocate multiple master/slave groups.

\section{APIs for Inter-Core Time Aggregation Scheduler (IAS)}

In this section, we propose the APIs for IAS, set_ias_agg and set_ias_alloc, which deal with the problems described in Section 2.4. In Section 3.1, we explain set_ias_agg, which controls the strength of aggregation of sibling threads. In Section 3.2, we explain set_ias_alloc, which controls the allocation of master/slave.

\section{1 set_ias_agg}

There are five arguments passed to set_ias_agg as shown below.

$$
\begin{aligned}
& \text { - pid } \\
& \text { - agg } \\
& \text { - bonus_type }
\end{aligned}
$$


- bonus_value

- limit

We specify the process ID to control the aggregation of its sibling threads by pid and set_ias_agg sets the values of agg, bonus_type, bonus_value, and limit to the members added in the memory address space of thread pid. The values stored in the members in the memory address spaces are the parameters for IAS to make scheduling decisions. We explain each member below.

agg must be 0 or 1 . If agg is 0 , IAS does not aggregate sibling threads of pid. If $a g g$ is 1, IAS aggregates sibling threads of pid. The kernel initializes the values of $a g g$ as 0 and IAS does not aggregate any threads by default. Users should set agg as 1 only when they consider that the aggregation of the sibling threads is effective.

IAS provides two ways to specify the priority bonus with bonus_type and bonus_value. bonus_type takes 0 or 1 . If bonus_type is 0 , IAS gives the priority bonus in vruntime specified in bonus_value. In this case, bonus_value ranges from 0 to over 18,446,744,073G vruntime ${ }^{2}$. If bonus_type is 1 , IAS gives the priority bonus by multiplying the quantum time of the previously executed thread by bonus_value. There are four reasons to utilize the quantum time of previously executed thread. Firstly, the change of the additional vruntime influences the order of threads in the runqueues. We assume that parallel tasks are equally assigned to sibling threads during their execution. In this case, the difference of vruntime between sibling threads are less than the quantum time of previously executed thread. For this reason, we consider that setting the quantum time as the criterion of the priority bonus is reasonable. Secondly, the quantum time changes dynamically according to the workload, therefore, it is hard for users to statically guess the effective priority bonus. Thirdly, it is easy to calculate the quantum time because CFS tracks it for the calculation of vruntime. Fourthly, it is easier to make a guideline of using IAS between different programs. As we mention, the range of bonus_value is too wide to properly set the effective priority bonus to enhance the throughput while keeping a certain fairness between different programs. For these reasons, we consider that using the quantum time provides a proper way of the abstraction.

Users can also restrict the number of aggregations on a single Core by specifying the value of limit. IAS counts the number of aggregations on a single Core, and IAS does not give the priority bonus to sibling threads when the number of aggregations exceeds limit.

\section{2 set_ias_alloc}

set_ias_alloc allocates master/slave roles to each Core. The arguments passed to set_ias_alloc are numbers which specify the role of each Core. We assume the use of set_ias_alloc from command lines because the allocation of

\footnotetext{
${ }^{2}$ vruntime has the type of unsigned long long and we assume to use 32 bit kernel here.
} 
master/slave influences the execution of all threads in the system and we need to observe the situation before running programs.

IAS controls the role of each Core by using an array ias_job_alloc [], which we defined inside the kernel. The index of ias_job_alloc [] corresponds to the ID of Core starting with 0. For example, the role of Core 2 is stored in ias_job_alloc [2]. So far, IAS is able to deal with octa-core processors and the role of each Core is specified with numbers from 0 to 7 . We show the correspondence between the numbers and its role in Table 2. In Table 2, Cores on slave_0 follow the aggregation of Core on master_0. Following command sets two inter-core aggregations on a quad-core processor, one inter-core aggregation between Core 0 and 1 and another between Core 2 and 3.

\$ set_ias_alloc 0123

Table 2. The correspondence between the number and its role in set_ias_alloc

\begin{tabular}{c|l}
\hline Number in ias_job_alloc [] & Correspondent Role \\
\hline \hline 0 & master_0 \\
\hline 1 & slave_0 \\
\hline 2 & master_1 \\
\hline 3 & slave_1 \\
\hline 4 & master_2 \\
\hline 5 & slave_2 \\
\hline 6 & master_3 \\
\hline 7 & slave_3
\end{tabular}

\section{Evaluation of APIs with memory program in SysBench}

In this section, we evaluate the effectiveness of APIs with memory program in SysBench[14]. In Section 4.1, we explain memory program, our experimental platform, and the method of the evaluation. In Section 4.2, we explain the result and show that our API is effective in utilizing IAS.

\section{1 memory Program and Experimental Platform}

SysBench benchmark suites is a collection of benchmark programs to evaluate the performance of workloads related to Online Transaction Processing. memory program in SysBench focuses on the performance of sequential reads from or writes to a memory block. memory program creates sibling threads and lets them repeat accessing a specified size of shared or unique memory block until the total accessed size exceeds a user-specified size. There are several metrics in memory program such as the average time of each data access and the total elapsed time. 
Table 3. Parameters for evaluating memory program

\begin{tabular}{|c|c|c|}
\hline \multicolumn{2}{|c|}{ Parameter } & Specified value \\
\hline \multicolumn{2}{|l|}{-num-threads } & 100 \\
\hline \multicolumn{2}{|l|}{-memory-oper } & write \\
\hline \multicolumn{2}{|l|}{-memory-scope } & global \\
\hline \multirow[t]{2}{*}{-memory-block-size } & set_ias_agg & $4(\mathrm{MB})$ \\
\hline & set_ias_alloc & $\begin{array}{l}1,2,4,6,8,10 \\
12,14,16(\mathrm{MB}) \\
\end{array}$ \\
\hline \multirow[t]{2}{*}{-memory-total-size } & set_ias_agg & $10(\mathrm{~GB})$ \\
\hline & set_ias_alloc & $\begin{array}{l}5,10,15 \\
20,25(\mathrm{~GB})\end{array}$ \\
\hline
\end{tabular}

We can control memory program through the parameters such as the number of threads, the size of the memory block, the total access size.

We show the parameters used for the evaluation in Table 3 . In the following explanation, we show the parameter used in the parentheses. We execute 10 memory programs simultaneously to mingle threads of different memory address spaces. We let each program create 100 sibling threads (-num-threads $=100)$ and let sibling threads access the shared memory block (-memory-scope=global) to focus on the effect of utilizing the locality between sibling threads. Each thread writes to the memory block sequentially $(-$ memory-oper=write). We can control the size of the memory block (-memory-block-size) and the total access size (-memory-total-size). We use different values for -memory-block-size and memory-total-size in the evaluation of each API and explain them in the method of the evaluation below.

We also show our experimental platform in Table 4. Intel Core 2 Quad is quad-core processor and has two L2 caches, each of which is shared by two Cores.

Table 4. Specification of our experimental platform

\begin{tabular}{l|l}
\hline \hline Processor & Intel Core 2 Quad \\
\hline L2 Cache Size / Latency & $3 \mathrm{MB} \times 2 / 5.6 \mathrm{~ns}$ \\
\hline Memory Size / Latency & $1.8 \mathrm{~GB} / 74.4 \mathrm{~ns}$ \\
\hline OS / kernel & CentOS 5.3 / Linux 2.6.24 \\
\hline
\end{tabular}

We measure the total elapsed time and the number of resource stalls (RESOURCE_STALLS.ANY[15]), and compare the results in CFS and IAS with APIs. We show the method of the evaluation in each API below. 
Evaluation Method of set_ias_agg In the evaluation of set_ias_agg, we focus on the function of setting the value of the priority bonus. We use a single value for the -memory-block-size and the -memory-total-size as shown in Table 3 and set the same parameter to 10 memory programs. We compare the result from the different methods of setting the priority bonus, directly specifying it in vruntime or multiplying the quantum time of previously executed thread. In case of setting bonus_type as 0 , we try broad range of bonus_value from $1 \mathrm{~K}$ to $10 \mathrm{M}$ vruntime because it is difficult to guess the effective value. In case of setting bonus_type as 1 , we multiply the quantum time by from 1 to 5 .

Evaluation Method of set_ias_alloc In the evaluation of set_ias_alloc, we use five different -memory-total-size values for 10 memory programs as shown in Table 3, assuming a situation when a user executes several different programs. We also change -memory-block-size to investigate the range of the effect of IAS in setting multiple master/slave groups.

We prepare three cases, where we set different master/slave groups and processor affinity of the threads, and compare their results. The first case is to use a single master/slave group, where Core 0 is master_ 0 and other three Cores are slave_0, and not to set the processor affinity to any threads (Case 1 ). We set two master/slave groups, where Core 0 is master_ 0 and Core 1 is slave_0 while Core 2 is master_ 1 and Core 3 is slave_ 1 , in the second and the third case (Case 2 and Case 3). The difference between Case 2 and Case 3 is the setting of the processor affinity of threads. In Case 2, we do not specify the processor affinity of threads and threads can be executed in every Core. In Case 3 , we divide memory programs into two groups as programs of the same total size are split into different master/slave group. For example, sibling threads of a memory program with -memory-total-size of 10GB are executed on Core 0 and Core 1 while sibling threads of another memory program with -memory-total-size of 10GB are executed on Core 2 and Core 3 . By specifying the processor affinity above, we can divide the workload equally into two Core groups with different L2 caches and restrict the overhead of communication between Cores. We expect the optimal performance in Case 3 and evaluate how close the result in Case 1 and 2 will be. We set the priority bonus as 50M vruntime based on our previous experiment[7].

\subsection{Results}

In this section, we firstly show the results of the evaluation of set_ias_agg. Succeedingly, we show the results of the evaluation of set_ias_alloc.

Results of the evaluation of set_ias_agg We show the result of the evaluation of set_ias_agg in Fig. 4. In Fig. 4, we show the ratio of the execution time in IAS against CFS (lines), and the absolute value of the resource stalls (bars) in each parameter. In Fig. 4, we express each parameter as d_ $[1,2,3,4,5]$ when we set bonus_type as 1 , and $\mathbf{s}_{-}[1 \mathrm{~K}, 10 \mathrm{~K}, 100 \mathrm{~K}, 1 \mathrm{M}, 10 \mathrm{M}]$ when we set bonus_type 
as 0 . We see that the reduction of the execution time and the resource stalls becomes larger as we increase the value of the parameter when we set bonus_type as 1 . On the other hand, we see little effect of IAS when bonus_value is from $1 \mathrm{~K}$ to $100 \mathrm{~K}$ when we set bonus_type as 0 . When we set bonus_value higher than $1 \mathrm{M}$ vruntime, we see the effect becomes larger. We consider that bonus_value below $1 \mathrm{M}$ vruntime is too small in this experiment because the average additional vruntime, which we measure simultaneously during the experiment, is $33 \mathrm{M}$.

We conclude that we can set the priority bonus easily and effectively by setting the priority bonus based on the quantum time rather than specifying in vruntime.

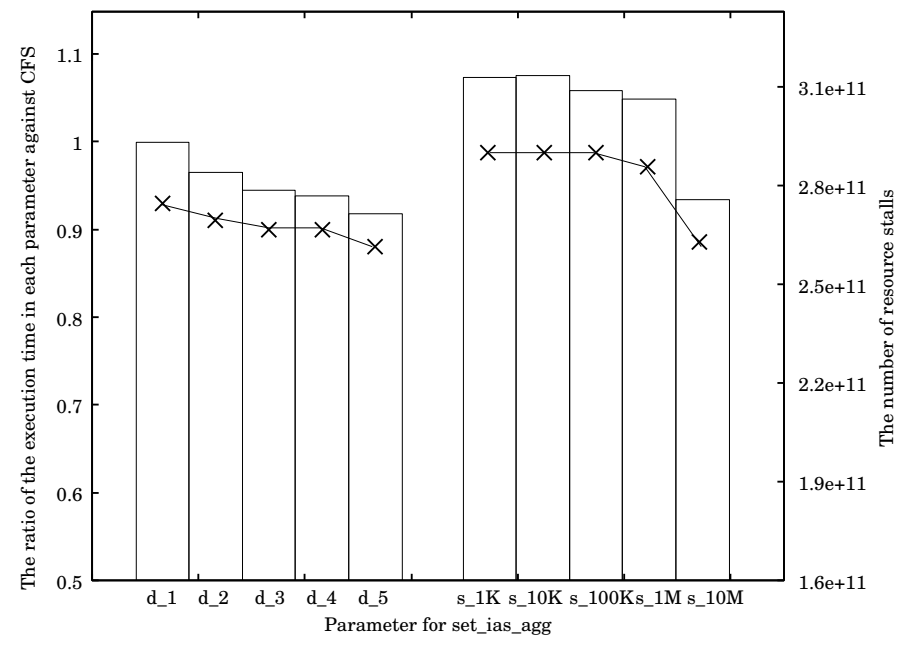

Fig. 4. The effect on the execution time (lines) and the resource stalls (bars) in using set_ias_agg

Results of the evaluation of set_ias_alloc We show the result of the evaluation of set_ias_alloc in Fig. 5. In Fig. 5, we show the ratio of the execution time in IAS against CFS in Case 1, 2, 3. We can see the effect in Case 2 and 3 are larger than that in Case 1 . We consider that the result shows the effect of space-multiplexing, which reduces the overhead of communication between Cores in Case 2 and 3. We also consider that the effect will be larger in manycore processors with deeper memory hierarchy.

When we compare Case 2 and Case 3, Case 3 is advantageous only when the memory block is less than $6 \mathrm{MB}$. In other parameters, the effects in Case 2 and 3 are almost the same while Case 3 is the optimal setting as we described in Section 4.1. Considering users do not have to set the processor affinity, Case 2 becomes more advantageous as working set gets larger. We conclude that we 
can gain the effect of reducing the overhead of communication between Cores by setting multiple master/slave Cores with set_ias_alloc.

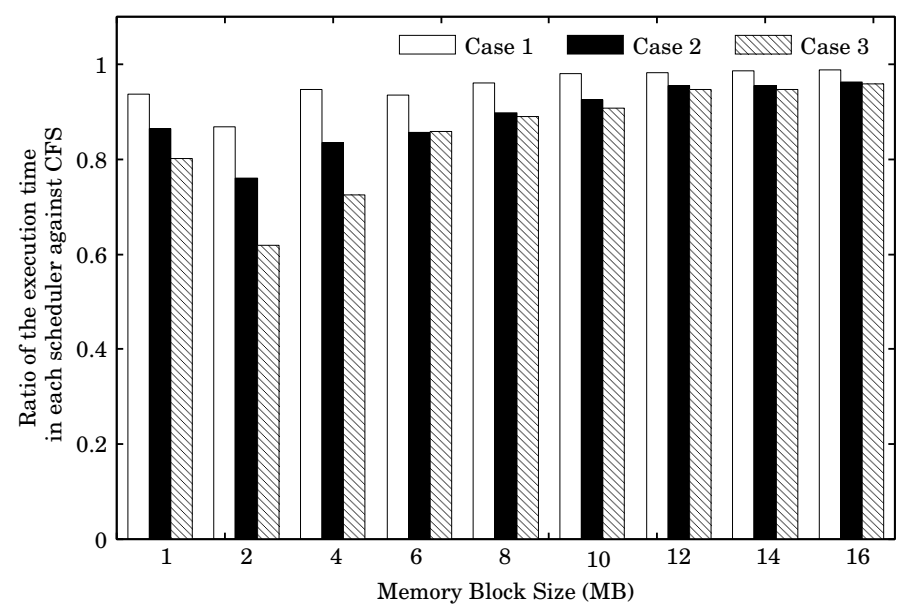

Fig. 5. The effect on the execution time in setting multiple ia_mm with set_ias_agg

\section{Related Research}

As caches are generally shared between Cores in multi-core processors, many thread-level schedulers have been proposed to utilize the caches. Many researches proposed to split the thread execution into the sampling phase and the scheduling phase[16-18]. In the sampling phase, the kernel samples the information of each thread execution. In the scheduling phase, the kernel schedules the combination of threads to execute simultaneously between different Cores based on the information obtained in the sampling phase. For example, Fedorova[18] calculates the size of the working set of each thread by tracing its behavior in the sampling phase. They schedule the combinations of threads to let the sum of the working set fit within the capacity of the L2 cache. The benefit of sampling and scheduling approach is that we can apply this method to any case of thread execution in theory. The problem of this approach is the overhead of sampling information, especially when running many threads, as IAS supposes[12, 19]. Moreover, the complexity of optimal co-scheduling in multi-core processor, where a cache is shared between all Cores and the number of Cores is more than 2 , is NP-complete[2]. We focus on a more realistic approach. Even though IAS does not intend to schedule threads optimally, IAS only focuses on the memory address space of each thread and its overhead is little as we see in Section 2.3.

The basic idea of our approach is similar to that of Chen[3] in that their scheduling algorithm executes threads sharing the working set simultaneously 
on different Cores to utilize the shared cache. Chen also proposes a compiler to control the granularity of threads to fit with the caches of the processor. Chen's approach is applicable to fine-grained multi-threaded programs, which contains DAGs inside, and shows that their scheduling method can enhance the throughput by carefully tuning the granularity of threads by their compiler. The difference between IAS and Chen's approach is that IAS is intended to work for multi-programmed execution while Chen only considers single-programmed execution. IAS does not detect the size of the working set shared between sibling threads while Chen's approach does not consider the influence from other programs. We consider that we can enhance the performance of broader range of multi-threaded programs by mixing IAS and Chen's approach.

Ziemba also focuses on the locality of references between sibling threads and investigates the effect of space-multiplexing with a Web application server[20]. They set different processor affinities for threads of HTTP and application servers in executing SPECweb benchmark[21]. They present their aggregation is effective and enhances the performance of the Web application server, indicating the locality of references between sibling threads. However, they mention that it is difficult to statically analyze applications and optimally set processor affinities. In this paper, we present that we can gain the effect of space-multiplexing without setting processor affinities in each thread.

\section{Conclusion}

This paper proposes and evaluates APIs for IAS, which is a kernel-level thread scheduler to enhance the performance of multi-threaded programs. We have proposed IAS, which dynamically aggregates sibling threads in $O(1)$ to utilize the cache shared between Cores. In this paper, we present two APIs, set_ias_agg, which controls the aggregation of sibling threads, and set_ias_alloc, which controls master/slave groups. The effectiveness of our API is described in two aspects. Firstly, we show that we can effectively and easily set the aggregation strength in IAS based on the quantum time of the previously executed thread by using API set_ias_agg. Secondly, we show that we can gain the effect of space-multiplexing by splitting Cores into several groups and running IAS per group without setting the processor affinity of each thread by using API set_ias_alloc.

Our future work includes the investigation of the effect of IAS with more general benchmark applications. We consider that IAS is especially effective in a benchmark application such as SPECweb[21], which runs multiple multithreaded programs simultaneously. We also investigate the efficiency of Helperthread mentioned in Section 1. Even though we can set the priority bonus easily with set_ias_agg, we still have to set the parameter manually. We will develop Helper-thread mechanism to detect the degradation of multi-threaded programs and automatically tune the priority bonuses to enhance the effect of IAS. In addition, we will develop scheduling strategies to control the behavior of Helper- 
thread such as the frequency of sampling thread information and the granularity of parameter changes.

\section{References}

1. Kim, S., et al.,: Fair Cache Sharing and Partitioning in a Chip Multiprocessor Architecture, Proceedings of the 13th International Conference on Parallel Architectures and Compilation Techniques, pp.111-122 (2004)

2. Jiang, Y., et al.,: Analysis and Approximation of Optimal Co-Scheduling on Chip Multiprocessors, Proceedings of the 17th International Conference on Parallel Architectures and Compilation Techniques, pp.220-229 (2008)

3. Chen, S., et al., Scheduling Threads for Constructive Cache Sharing on CMPs, Proceedings of 19th ACM symposium on Parallel Algorithms and Architectures, pp.105-115, (2007)

4. DaCapo benchmark suite, http://dacapobench.org/

5. The PARSEC Benchmark Suite, http://parsec.cs.princeton.edu/

6. Chishti, Z., et al.,: Optimizing Replication, Communication, and Capacity Allocation in CMPs, Proceedings of the 32nd International Symposium on Computer Architecture, pp.357-368 (2005)

7. Yamada, S., et al.,: Development of a Thread Scheduler for Global Aggregation of Sibling Threads, Research Reports on Information Science and Electrical Engineering of Kyushu University, Vol. 1, No. 2, pp.69-74 (2008)

8. Yamada, S., et al.,: Impact of Priority Bonuses of Inter-Core Aggregation Scheduler on a Commodity CMP Platform, Workshop on Managed Many-Core Systems (MMCS) co-located with ASPLOS'09 http://www.cercs.gatech.edu/mmcs09/program.htm (2009)

9. RUBiS: Rice University Bidding System, http://rubis.ow2.org/

10. K. Keeton, et al.,: Performance Characterization of a Quad Pentium Pro SMP Using OLTP Workloads, Proceedings of the 25th annual International Symposium on Computer Architecture, pp.15-26 (1998)

11. J. Redstone, et al.,: An Analysis of Operating System Behavior on a Simultaneous Multithreaded Architecture, Proceedings of the 9th International Conference on Architectural Support for Programming Languages and Operating Systems, pp.245-256 (2000)

12. DeVuyst, M., et al.,: Exploiting Unbalanced Thread Scheduling for Energy and Performance on a CMP of SMT Processors, Proceedings of 20th IEEE International Parallel \& Distributed Processing Symposium (2006)

13. Yamada, S., et al.,: Effect of Context Aware Scheduler on TLB, Workshop on Multi-Threaded Architectures and Applications, Published in CD (2008)

14. SysBench: a system performance benchmark, http://sysbench.sourceforge.net/

15. Intel 64 and IA-32 Architectures Software Developer's Man ual, Volume 3B: System Programming Guide, Part 2, http://www.intel.com/products/processor/manuals/index.htm

16. Parekh, S., et al.,: Thread-Sensitive Scheduling for SMT Processors, Technical report, Dept. of Computer Science and Engineering, University of Washington (2000)

17. Snavely, A., et al.,: Symbiotic Jobscheduling with Priorities for a Simultaneous Multithreading Processor, Proceedings of International Conference on Measurement and Modeling of Computer Systems, pp.66-76 (2002) 
18. Fedorova, A., et al.,: Performance of Multithreaded Chip Multiprocessors and Implications for Operating System Design, Proceedings of USENIX 2005 Annual Technical Conference, pp.395-398 (2005)

19. Chandra, D., et al.,: Predicting Inter-Thread Cache Contention on a Chip MultiProcessor Architecture, Proceedings of 11th International Symposium on HighPerformance Computer Architecture, pp.340 - 351 (2005)

20. Ziemba, S., et al.,: Analyzing the Effectiveness of Multicore Scheduling Using Performance Counters, Proceedings of Workshop on the Interaction between Operating Systems and Computer Architecture (2008)

21. SPECweb, http://www.spec.org/web2009/ 